\title{
ON THE DYNAMICS OF NON-STATIONARY STELLAR CLUSTER IN GALAXY
}

\section{T. S. Kozhanov}

Kazakh national agrarian university 480100, Almaty-100, ave. Abai 8,

\author{
K. A. Omarkulov
}

Kokshetau University, Republic of Kazakhstan

\begin{abstract}
We followed Wielen's (1974) and Osipkov's, Kozhanov's (1986) procedure to derive the equation of motion of a cluster in the solar neighborhood in taking into account of a galactic tidal field, which is assume to have axial symmetry, and gravitational influence of spherical stellar clouds.

On the basic of this equations and using the linear approximation for the galactic field (Wielen 1974) one can derive virial equation of Jacobi for variable masses of stellar clusters. The non-stability by Lagrange condition, describing behavior of this system is obtained.
\end{abstract}

Let us consider the stellar cluster with term mass, changing on time, in regular gravity field of Galaxy. This cluster is embedded into homogenous spherical stellar cloud in consideration that in initial moment the cloud center coincide with the mass center of cluster, which moves as a material point on circular orbit in Galaxy plain. We will assume, that on cluster affect: the intrinsic regular gravitational field; the regular gravitational field of Galaxy (in impulse approximation); the background, in which the cluster is embedded (for simplicity we will consider it as homogenous and isotropic).

The equations of motion of clusters stars in rotational coordinate system (Wielen 1974) at availability of gravitating background (Osipkov, Kozhanov 1986) have the form:

$$
\begin{array}{r}
\ddot{x}_{i}-2 \varpi_{c} \dot{y}_{i}+\left(\chi_{0}^{2}--\chi_{R}^{2}\right) x_{i}=-G \sum_{i \neq j}^{n} \frac{m_{i}(t)}{r_{i j}^{3}}\left(x_{j}-x_{i}\right), \\
\ddot{y}_{i}-2 \varpi_{c} \dot{x}_{i}+\chi_{0}^{2} y_{i}=-G \sum_{i \neq j}^{n} \frac{m_{i}(t)}{r_{i j}^{3}}\left(y_{j}-y_{i}\right), \\
\ddot{z}_{i}+\left(\chi_{0}^{2}-\chi_{z}^{2}\right) z_{i}=-G \sum_{i \neq j}^{n} \frac{m_{i}(t)}{r_{i j}^{3}}\left(z_{j}-z_{i}\right),
\end{array}
$$

where: $\varpi_{c}=(A-B)$ - the circular frequency of revolving of cluster center relatively the Galaxy center, $\chi_{R}^{2}=4 A(A-B), \chi_{z}=C$ - the frequency of small vertical oscillations of trial star in Galaxy, $A, B, C$-the Oort-Kuzmin parameters. The system of equations (1) at constant value of cluster terms mass has for $i$-th star the Jacobi integral:

$$
C_{i}=\frac{1}{2} \vartheta_{i}^{2}-G \sum_{i \neq j}^{n} \frac{m_{i}}{r_{j}-r_{i}}-\frac{1}{2}\left[\chi_{0}^{2}\left(x_{i}^{2}+y_{i}^{2}+z_{i}^{2}\right)+\chi_{R}^{2} x_{i}^{2}+\chi_{z}^{2} z_{i}^{2}\right]
$$

$\mathrm{C}_{i}$ - give the equipotential surfaces at zero velocity $\left(\vartheta_{i}=0\right)$.

The last closed equipotential surface passes throughout two collinear Lagrangian points $L_{1}$ and $L_{2}$. The relative Lagrangian points on axis $x$ from cluster center is:

$$
x_{L}=\left(\frac{G M}{\chi_{R}^{2}-\chi_{0}^{2}}\right)^{\frac{1}{3}}=\left(\frac{G M}{4 A \varpi_{c}-\chi_{0}^{2}}\right)^{\frac{1}{3}}
$$


The equation (3) at $\chi_{0}^{2}=0$ is named the tidal radius of cluster (King 1961). The critical value of Jacobi constant has the form

$$
C_{L x}=-\frac{3}{2}\left[G^{2} M^{2}\left(-\chi_{0}^{2}+4 A \varpi_{c}\right)\right]^{\frac{1}{3}} \text { hfill (4) }
$$

where $M$ - the total mass of cluster. From relation (3) it is seen, that the gravitating background increases the size of King tidal radius and decreases the absolute value $C_{L}$. Besides that we have the points of liberation on axes $y$ and $z$, i.e.

$$
y_{L}=-\left(\frac{G M}{\chi_{0}^{2}}\right)^{\frac{1}{3}}, z_{L}=\left(\frac{G M}{\chi_{z}^{2}-\chi_{0}^{2}}\right)^{\frac{1}{3}}
$$

Respectively, the total value of Jacobi integral has the form:

$$
C_{L}= \pm \frac{3}{2}(G M)^{\frac{3}{2}}\left[\left(4 A \varpi_{c}-\chi_{0}^{2}\right)^{\frac{2}{3}}+\left(\chi_{0}^{2}\right)^{\frac{2}{3}}+\left(\chi_{0}^{2}-C^{2}\right)^{\frac{2}{3}}\right]^{\frac{1}{2}} .
$$

According to the Edington -Jeans law for variable mass $\frac{d m}{d t}=-k m^{n}$ ( $k=$ const), at $n=1$ the Lagrange- Jacobi dynamical equation for system (1) have the form :

$$
\frac{d^{2} J}{d t^{2}}+2 \delta \frac{d J}{d t}+\Omega^{2} J=\frac{2}{k} \frac{d E}{d t}+6 E+2 \varpi_{c} L_{z}
$$

where $E=T+(-U)$ - the total energy of cluster, $L_{z}$ - the vertical component of major vector of moment of momentum, $q_{x}, q_{y}, q_{z}$ - - the "weighted" factors of cluster moment inertia, i.e.

$$
J_{x}=q_{x} J
$$

It stands to reason, their values are determined by cluster oblates. In particular, if the system has the spherical form, then $q_{x}=q_{y}=q_{z}=\frac{1}{3}$,

$$
\delta=\frac{k^{2}-\chi_{0}^{2}+q_{x} \chi_{R}^{2}+q_{z} \chi_{z}^{2}}{k}, \Omega^{2}=\chi_{0}^{2}-\chi_{R}^{2} q_{x}-\chi_{z}^{2} q_{z}-2 \varpi_{c}^{2}\left(q_{x}+q_{y}\right)+k^{2} .
$$

This equation coincides on exterior form with the damped oscillations of material point under exterior "constraining force" $P(t)$

$$
P(t)=6 E+\frac{2}{k} \frac{d E}{d t}+2 \varpi_{c} L_{z} .
$$

The postulation: if for the Oort-Kuzmin parameters are performed the inequality:

$$
\delta \text { or }\left(k^{2}+q_{x} \chi_{R}^{2}+q_{z} \chi_{z}^{2}\right) \chi_{0}^{2}
$$

then the rotating stellar cluster on gravitating background is in non-stable state, i.e. at least the one form mutual distances between stars aim to infinity.

The general solution of equation (7) has the following form:

$$
J(t)=D_{1} e^{n_{1} t}+D_{2} e^{n_{2} t}+\frac{1}{2 \sqrt{\delta^{2}-\Omega^{2}}} e^{n_{1} t} \int e^{-n_{2} t} p(t) d t-\frac{1}{2 \sqrt{\delta^{2}-\Omega^{2}}} e^{n_{2} t} \int e^{-n_{1} t} p(t) d t
$$

where $D_{1}, D_{2}$ - the constants of integration,

$$
n_{1}=-\delta+\sqrt{\delta^{2}-\Omega^{2}}, n_{2}=-\delta-\sqrt{\delta^{2}-\Omega^{2}}
$$

Thus, at accomplishing of condition (11) $n_{1}$, i.e. at $t \rightarrow \infty, e^{n_{1} t}$ aim to infinity; this means that the system moment of inertia indefinitely rise with time.

It may establish the different equilibrium states of cluster versus the Oort-Kuzmin parameters and relative $\chi_{0}^{2}$ and $k$. Such investigations will be made in future.

\section{References}

Osipkov L.P., Kozhanov T.S. 1986. In: Stationary and non-stationary gravitating systems. Almaty, Nauka. P. 14-18. (in Russian)

Wielen R., 1974. In Proceedings of the first Europ. Astron. Meeting, L.N. 2.P.236

King I.R., 1962. Astr. J. V.67, $N$ 8. P.471. 